\title{
Signatures of a staggered-flux phase in the $t-J$ model with two holes on a 32-site lattice
}

\author{
P. W. Leung* \\ Department of Physics, Hong Kong University of Science and Technology, Clear Water Bay, Hong Kong, China
}

(Received 15 March 2000)

\begin{abstract}
We study the relevance of the staggered-flux phase in the $t-J$ model using a system with two holes on a 32-site lattice with periodic boundary conditions. We find a staggered-flux pattern in the current-current correlation in the lowest energy $d$-wave state where there is mutual attraction between the holes. This staggered correlation decays faster with distance when the hole binding becomes stronger. This is in complete agreement with a recent study by Ivanov, Lee, and Wen [Phys. Rev. Lett. 84, 3958 (2000)] based on the SU(2) theory, and strongly suggests that the staggered-flux phase is a key ingredient in the $t-J$ model. We further show that this staggered-flux pattern does not exist in a state where the holes repel each other. Correlations of the chirality operator $\mathbf{S}_{1} \cdot\left(\mathbf{S}_{2} \times \mathbf{S}_{3}\right)$ show that the staggered pattern of the chirality is closely tied to the holes.
\end{abstract}

The $t-J$ model is one of the most important microscopic models in the study of high-temperature superconductivity. It describes a doped $\mathrm{CuO}_{2}$ plane as a system of holes (or Zhang-Rice singlets ${ }^{1}$ ) moving in a spin background described by the antiferromagnetic Heisenberg model. The Hamiltonian is

$$
\mathcal{H}=-t \sum_{\langle i j\rangle \sigma}\left(\tilde{c}_{i \sigma}^{\dagger} \tilde{c}_{j \sigma}+\text { H.c. }\right)+J \sum_{\langle i j\rangle}\left(\mathbf{S}_{i} \cdot \mathbf{S}_{j}-\frac{1}{4} n_{i} n_{j}\right),
$$

where $\tilde{c}_{i \sigma}^{\dagger}$ and $\tilde{c}_{i \sigma}$ are the projected fermion operators, and $n_{i} \equiv \tilde{c}_{i \sigma}^{\dagger} \tilde{c}_{i \sigma}$ is the fermion number operator. Understanding the properties of this model is a major challenge in the theoretical study of high-temperature superconductivity. In spite of the simple form of $\mathcal{H}$, solving the $t-J$ model is nontrivial due to the strong interaction of the fermion objects. Meanfield theory solutions to the model often involve a fictitious statistical flux. ${ }^{2}$ The flux pattern in these "flux phases" can be uniform or staggered. In particular, it has been proposed that the staggered-flux phase in the $t$ - $J$ model might lower the energy of the system and become the ground state at parameters relevant to experimental systems. ${ }^{3}$ However, being an abstract mathematical quantity, it is difficult to find a suitable signature for the flux. Consequently, independent confirmation of the existence of flux phases by numerical or experimental studies is difficult. A previous attempt to search for flux phases using exact diagonalization on small clusters ${ }^{4}$ has been either negative or inconclusive.

As pointed out by Ivanov, Lee, and Wen, ${ }^{5}$ in the case of the doped $t-J$ model a signature for the staggered-flux phase can be found in the current-current correlation. Using a Gutzwiller-projected $d$-wave pairing wave function, they found such a pattern in the current correlation; namely, the hole current goes around the elementary square plaquettes in the counterclockwise and clockwise directions, suggestive of positive and negative fluxes through the plaquettes in a staggered manner. They explained this observation by showing that the Gutzwiller-projected $d$-wave pairing wave function is equivalent to the $\mathrm{SU}(2)$ projected staggered-flux wave function. Although this result shows that the concept of a staggered-flux phase is relevant in the projected $d$-wave pairing wave function, it does not answer the question of whether this phase exists in the $t-J$ model. The origin of the staggered-flux pattern in their study is in the staggered-flux phase of the $\mathrm{SU}(2)$ projected wave function. It will be very interesting to check whether such a pattern in the current correlation exists using an approach that is independent of the mean-field theory.

Motivated by this result, we search for signatures for the staggered-flux phase in the $t-J$ model using exact diagonalization. This method is free from any analytical or numerical approximations, and thus can serve as an independent test for the result in Ref. 5. One serious drawback of exact diagonalization is that it is subjected to finite-size effect. This is particularly serious when we study correlation functions where the size and boundary conditions can have significant effects. To overcome the finite-size effect as much as possible, we use the largest cluster on which the $t-J$ model is currently solvable by exact diagonalization, namely, the 32-site cluster with periodic boundary conditions. In order to study the current correlation, we need to dope the system with at least two holes. Such a two-hole $t$ - $J$ model on a 32 -site cluster has recently been solved using exact diagonalization. ${ }^{6,7}$ In this study we use the same lowest-energy $d$-wave states at different $J / t$ as in Ref. 7. They have $d_{x^{2}-y^{2}}$ symmetry and are the respective ground states when $J / t$ is not too small. Our primary interest is in the case $J / t=0.3$ which is believed to be an appropriate value to describe the doped cuprates. With this parameter, the $d$-wave state is a weak two-hole bound state - in the sense that it has a negative but small two-hole binding energy $E_{b} / t=-0.05146$. The root-mean-square separation of the two holes is $\sqrt{\left\langle r^{2}\right\rangle}=2.0587$. Note that this value does not imply that the holes are tightly bound. Due to the finite system size, two uncorrelated holes on this cluster will have $\sqrt{\left\langle r^{2}\right\rangle}=2.3827$.

Once the desired wave function is found, it is straightforward to evaluate the current correlation $\left\langle j_{k l} j_{m n}\right\rangle$, where the current on a bond linking the sites $k$ and $l$ is defined by

$$
j_{k l}=i t\left(\tilde{c}_{k \sigma}^{\dagger} \tilde{c}_{l \sigma}-\tilde{c}_{l \sigma}^{\dagger} \tilde{c}_{k \sigma}\right) .
$$

Following the notation of Ref. 5, we display the current correlation divided by the hole concentration, $\left\langle j_{k l} j_{m n}\right\rangle / x$. The result for $J / t=0.3$ is shown in Fig. 1. It is to be compared 


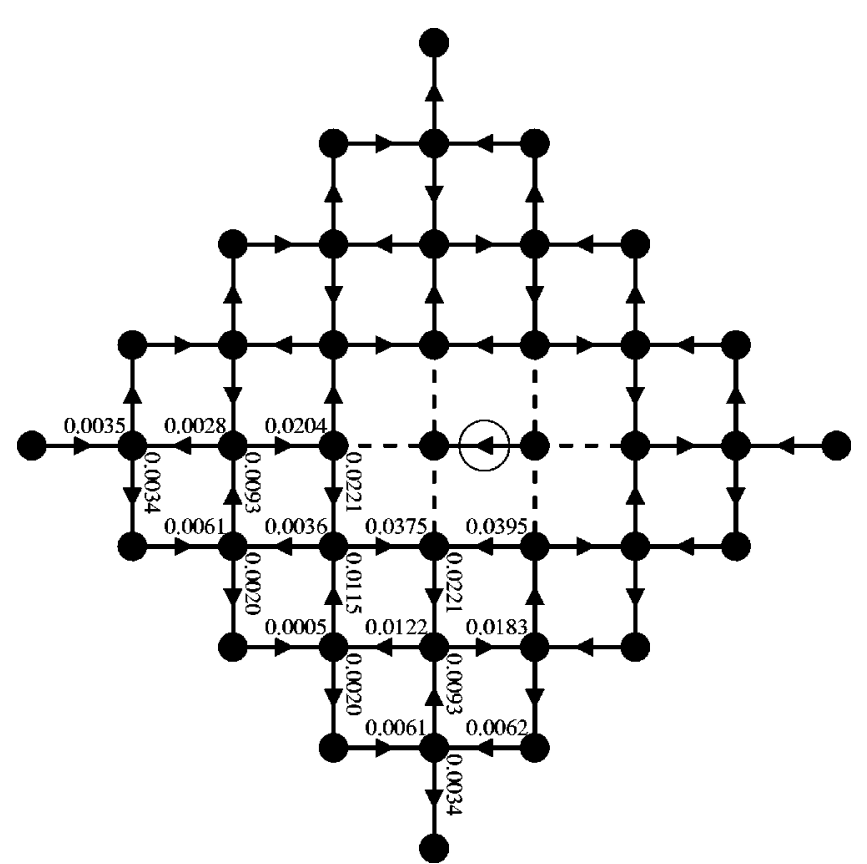

FIG. 1. The current correlation $\left\langle j_{k l} j_{m n}\right\rangle / x$ in the two-hole $t-J$ model at $J / t=0.3 . x \equiv 1 / 16$ is the hole concentration. The reference bond is indicated by an arrow with a circle. Arrows on other bonds point along the directions of positive correlation. Those arrows without numbers attached can be related by symmetry to those that have.

with Fig. 1 of Ref. 5. We note the very good agreement between these two results: not only all (except one) directions of the correlation agree, but also the corresponding numbers agree to the same order of magnitude. Given that the two results are obtained by very different approaches, their agreement is very surprising. The only exception to the agreement in the direction of the correlation is the one on the opposite side of the same square plaquette as the reference bond. This can be explained by a feature in the hole-hole correlation function,

$$
C_{h h}(r) \equiv\left\langle\left(1-n_{r}\right)\left(1-n_{0}\right)\right\rangle,
$$

of the $d$-wave state that we use. While $C_{h h}(1)>C_{h h}(\sqrt{2})$ in the projected $d$-wave pairing state, ${ }^{8}$ the opposite is true in our $d$-wave state. In fact the holes in our $d$-wave state have the largest probability to be at a distance $\sqrt{2}$ apart. ${ }^{9}$ When the holes are at the opposite vertices of the same plaquette, the hole current along the two opposite sides of the plaquette should be along the same direction.

In order to display the staggered pattern of the current correlation more clearly, we follow Ref. 5 and define the vorticity $V(\mathbf{r})$ of a square plaquette centered at $\mathbf{r}$ by summing up the currents around it in the counterclockwise direction. The vorticity-vorticity correlation divided by the hole concentration,

$$
C_{V V}(r) \equiv\langle V(r) V(0)\rangle / x,
$$

is shown in Fig. 2(a). Note that $C_{V V}$ can be computed from the current correlation, i.e., Fig. 2(a) is just another way of presenting the same data in Fig. 1. Figure 2(a) is to be compared to Fig. 1(b) of Ref. 5. They all have the same staggered pattern. Note that this staggered pattern has a $\pi$ phase shift,

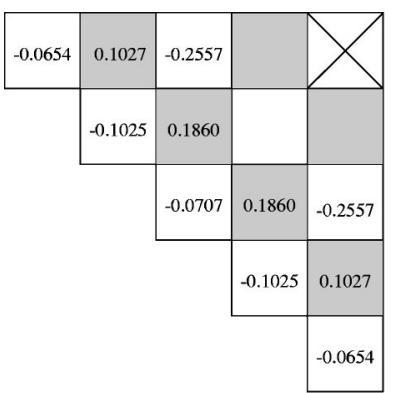

(a)

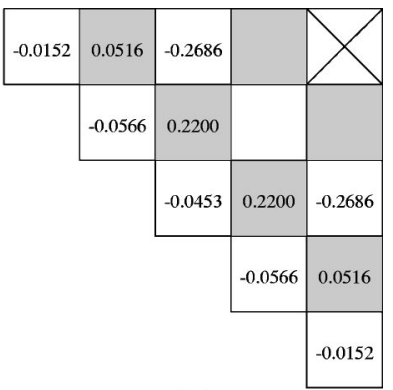

(b)
FIG. 2. The vorticity correlation $C_{V V}(r)$ at (a) $J / t=0.3$ and (b) $J / t=0.8$. The reference plaquette at $r=0$ is indicated by a cross inside it. Only part of the cluster is shown.

i.e., the sign of $\langle V(r) V(0)\rangle$ is $(-1)^{r_{x}+r_{y}+1}$. This $\pi$ phase shift was explained within the $\mathrm{SU}(2)$ picture in Ref. 5 by assuming the pairing of holes of opposite vorticities, i.e., circulating in opposite directions.

Since the hole current results from the motion of the holes, it is intuitively clear that the correlation between the current in different bonds must be related to the correlation between the holes in the corresponding locations. Therefore, in addition to the above weak bound state at $J / t=0.3$, we also study the wave function with the same symmetry but at $J / t=0.8$ where the holes are more tightly bound with $E_{b} / t$ $=-0.44423$ and $\sqrt{\left\langle r^{2}\right\rangle}=1.5120$. The purpose is to study how the current correlation changes when the binding of the holes is stronger. In Fig. 2(b) we show the vorticity correlation at $J / t=0.8$. We note that it has the same staggered with a $\pi$ phase shift pattern as that at $J / t=0.3$. Since the holes are more tightly bound in the present case, we expect the current correlation, and therefore the vorticity correlation, to have a shorter range. This is indeed the case when we compare it with Fig. 2(a). $C_{V V}(r)$ decreases much faster in the present case.

In Ref. 5 it was observed that in the projected $d$-wave pairing state the hole-hole and vorticity-vorticity correlations decay with the same power law. In Fig. 3 we plot these two quantities in our $d$-wave states at $J / t=0.3$ and 0.8 . As noted before, ${ }^{5,8} C_{h h}(r)$ depends on whether 0 and $r$ are on the same sublattice, and if so, whether $r$ is an even $\left(r_{x}\right.$ and $r_{y}$ are even) or odd ( $r_{x}$ and $r_{y}$ are odd) site. Those on the same sublattice and odd sites are enhanced, while those on the same sublattice and even sites are suppressed relative to those on different sublattices. If we assume that the hole correlation decays with a power law, $C_{h h}(r) \approx 1 / r^{\alpha}$, then the best fit gives $\alpha \approx 1.15<2$ at $J / t=0.3$, making it an unbound state when the system size goes to infinity. Being a tightly bound state, the hole correlation at $J / t=0.8$ decays much faster than that at $J / t=0.3$ - the corresponding $\alpha \approx 3.04$ is significantly larger than 2 . The same trend is found for the vorticity correlation. Nevertheless, with the limited number of data points we are not able to say conclusively whether they decay with the same law, nor whether they decay with power laws at all.

The above results show that a staggered-flux pattern exists in our exact $d$-wave state where the hole correlation decays with distance. In Ref. 5 such a pattern is interpreted as the binding of holes of opposite vorticities in the SU(2) language. It is therefore interesting to see, using our exact nu- 


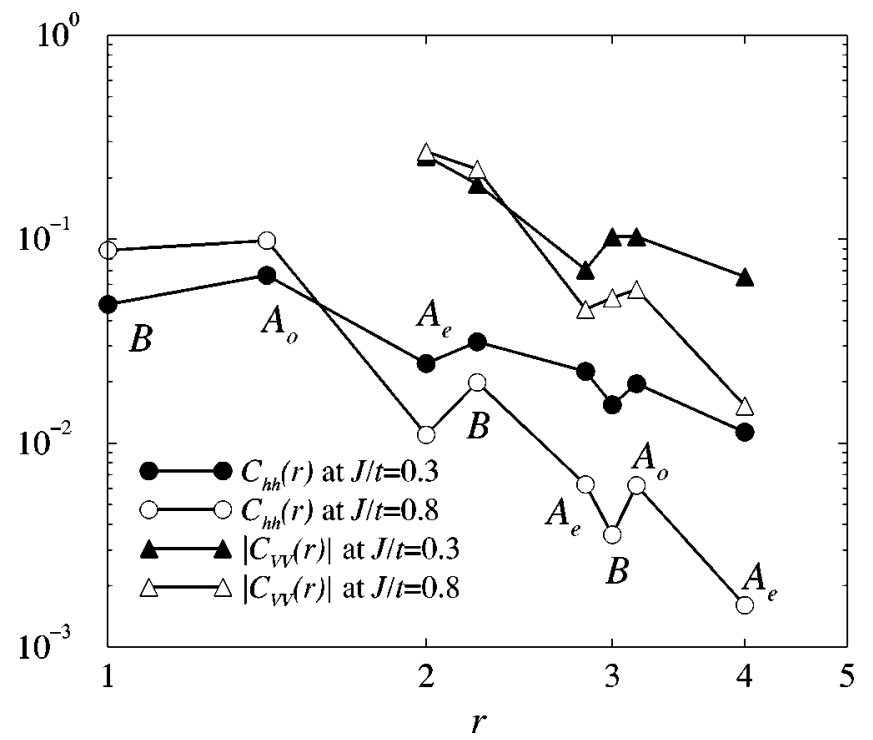

FIG. 3. The hole correlation $C_{h h}(r)$ and the vorticity correlation $\left|C_{V V}(r)\right|$ of the $d$-wave states at $J / t=0.3$ and 0.8. $A_{o}\left(A_{e}\right)$ indicates that the pair of holes at 0 and $r$ are on the same sublattice and $r$ is an odd (even) site. $B$ indicates that they are on opposite sublattices.

merical results, if such a pattern exists in a state where the holes repel. For this purpose we use a low-lying excited state of the same two-hole system at $J / t=0.3$. It is a doubly degenerate $p$-wave state with total momentum $(\pi, \pi)$ and is a spin singlet. ${ }^{10}$ Figure 4 shows the hole correlation of the $p_{y}$-wave state. In contrast to the $d$-wave ground state, the holes in this state repel each other $-C_{h h}$ increases with $r$ for small $r$. The mean-square separation between the holes is $\sqrt{\left\langle r^{2}\right\rangle}=2.5376$. Figure 5 shows the current correlations in the $p_{y}$-wave state. We observe that the magnitudes of the correlations are small even at small distances, and there is definitely no staggered-flux pattern. The same data plotted as vorticity correlation in Fig. 6 again confirm these observations. This is consistent with the interpretation that the staggered-flux pattern results from the pairing of the holes.

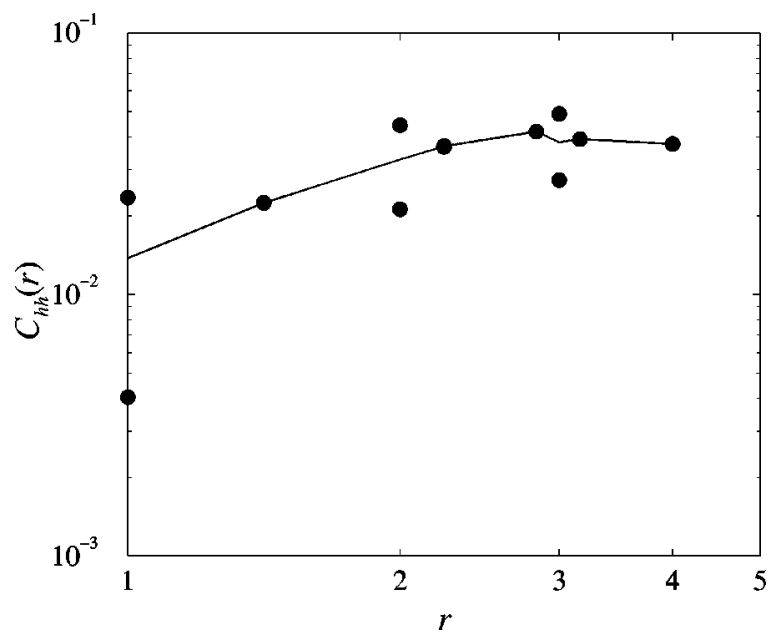

FIG. 4. The hole correlation $C_{h h}(r)$ of the $p_{y}$-wave state at $J / t=0.3$. Due to the lack of the rotational symmetry $C_{4}$ (i.e., a rotation by $\pi / 2$ ) in the wave function, there may be two inequivalent points at the same $r$. Their values are indicated by filled circles. The solid line joins their mean values.

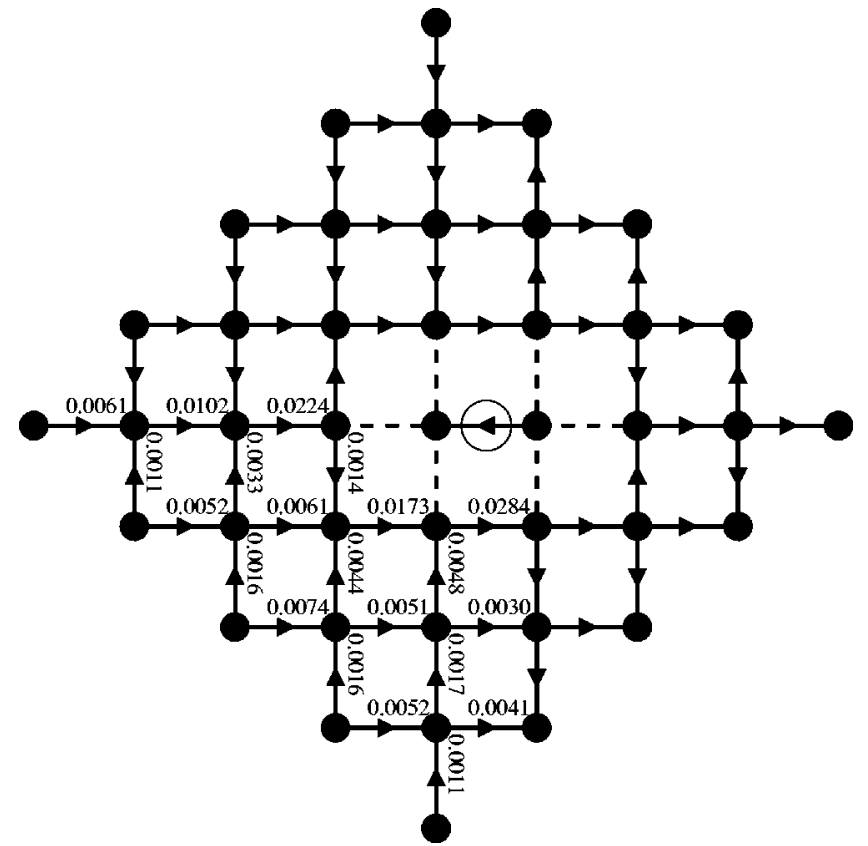

FIG. 5. Same as Fig. 1 but for the $p_{y}$-wave state. Due to the lack of the rotational symmetry $C_{4}$, there is an inequivalent correlation where the reference bond is a vertical one. It is not shown for the sake of simplicity.

We now return to the $d$-wave state at $J / t=0.3$ and search for another signature of the staggered-flux phase. It has been suggested that a physical interpretation of the statistical flux in the mean-field theory can be found in the spin chirality operator, $^{11}$

$$
\chi_{4}=n_{4} \mathbf{S}_{1} \cdot\left(\mathbf{S}_{2} \times \mathbf{S}_{3}\right),
$$

where $1,2,3$, and 4 are the vertices of a square plaquette in the counterclockwise direction. In Fig. 7 we plot the correlation $C_{\chi \chi}(r) \equiv\left\langle\chi_{r} \chi_{0}\right\rangle$ for the $d$-wave state at $J / t=0.3$. It is obvious that this correlation exhibits a short-range staggered pattern with a $\pi$ phase shift, but it decays very rapidly with distance. Since the chirality is related to a magnetic flux, which may in turn affect the movement of the holes, it is natural to associate the chiral order to the holes. To demonstrate the connection between the chirality and the hole current, we follow Ref. 12 and define the spin chirality operator around a hole,

$$
\chi_{4}^{h}=\left(1-n_{4}\right) \mathbf{S}_{1} \cdot\left(\mathbf{S}_{2} \times \mathbf{S}_{3}\right)
$$

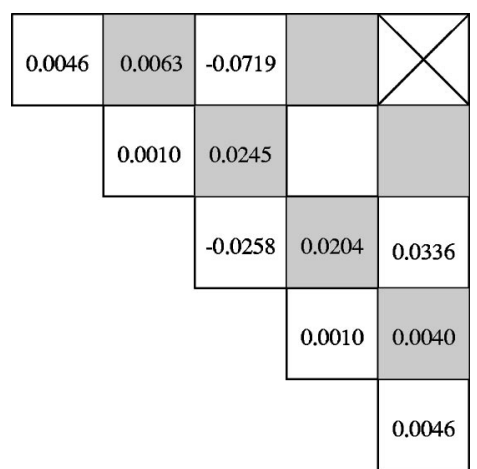

FIG. 6. The vorticity correlation $C_{V V}(r)$ of the $p_{y}$-wave state. 


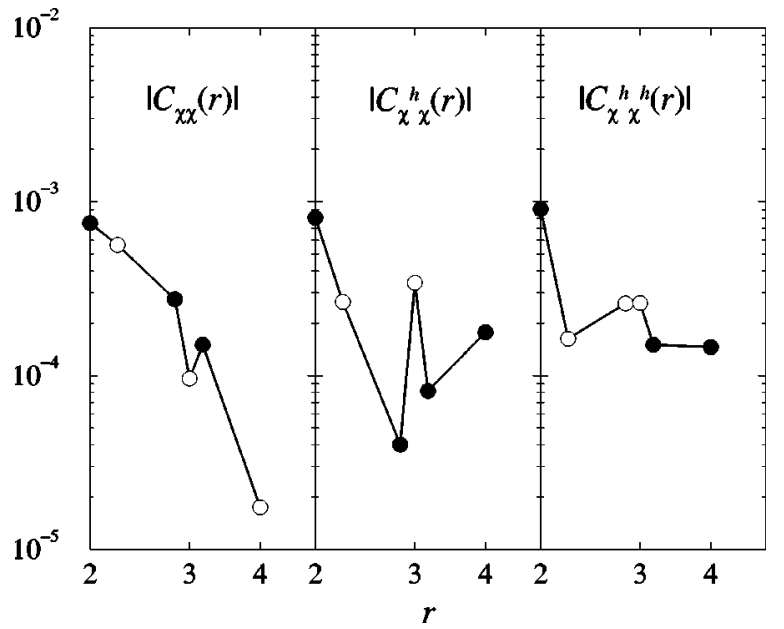

FIG. 7. Correlations of the chirality operators of the $d$-wave state at $J / t=0.3$ in the same logarithmic scale. Empty and solid circles represent positive and negative values, respectively.

The chiral order set up by a hole is displayed in the correlation $C_{\chi^{h} \chi}(r) \equiv\left\langle\chi_{r} \chi_{0}^{h}\right\rangle$ in Fig. 7. It has a staggered pattern with a $\pi$ phase shift. Compared to $C_{\chi \chi}(r)$, it decays slower, but has strong fluctuation as a function of $r$. Finally, we plot the correlation between the chirality around the two holes, $C_{\chi^{h} \chi^{h}}(r) \equiv\left\langle\chi_{r}^{h} \chi_{0}^{h}\right\rangle$. Its decay with distance is again much slower than in the case of $C_{\chi \chi}(r)$. These correlations show that the chiral order must be tied to the holes. Unfortunately, with the limited data we are not able to deduce conclusively

*Email address: P.W.Leung@ust.hk

${ }^{1}$ F. C. Zhang and T. M. Rice, Phys. Rev. B 37, 3759 (1988).

${ }^{2}$ For a review see P. A. Lee, in High Temperature Superconductivity Proceedings, Los Alamos, 1989, edited by K. S. Bedell et al. (Addison-Wesley, Redwood City, 1990).

${ }^{3}$ D. Poilblanc and Y. Hasegawa, Phys. Rev. B 41, 6989 (1990).

${ }^{4}$ D. Poilblanc, E. Dagotto, and J. Riera, Phys. Rev. B 43, 7899 (1991).

${ }^{5}$ D. A. Ivanov, P. A. Lee, and X.-G. Wen, Phys. Rev. Lett. 84, 3958 (2000).

${ }^{6}$ A. L. Chernyshev, P. W. Leung, and R. J. Gooding, J. Phys. whether long-range chiral order exists in the current system. We also note that similar results on the chirality correlations were obtained with the projected $d$-wave pairing wave function. $^{12}$

In conclusion, we have looked for signatures for the staggered-flux phase in the $t-J$ model by calculating the current and chirality correlations in the lowest-energy $d$-wave state of the two-hole $t-J$ model on a 32-site cluster with periodic boundary conditions. The current correlation clearly shows a staggered-flux pattern. Note that in the $d$-wave states at different $J / t$, the holes are mutually attractive $-C_{h h}(r)$ decays with distance. In this case the staggered correlation in the vorticity decays faster when the attraction between the holes becomes stronger. In the $p$-wave state where the holes repel $\left[C_{h h}(r)\right.$ increases with distance at small distances], no such staggered-flux pattern is found. Chirality correlations also show a similar staggered pattern. Although the numbers are small, they show that any long-range pattern in the chirality correlation must be related to the holes. Our results on the current correlation are consistent with a recent SU(2) study by Ivanov, Lee, and Wen. ${ }^{5}$ Being a completely independent approach, the fact that our unbiased numerical result agrees with theirs gives strong support to the notion that the staggered-flux phase is a key ingredient of the ground state in the $t-J$ model, at least in the low doping regime.

We are grateful to P. A. Lee for pointing out their work (Ref. 5) to us and for helpful discussions. This work was supported by the RGC of Hong Kong under Grant No. HKUST6144/97P.

Chem. Solids 59, 1814 (1998).

${ }^{7}$ A. L. Chernyshev, P. W. Leung, and R. J. Gooding, Phys. Rev. B 58, 13594 (1998).

${ }^{8}$ D. A. Ivanov, Ph.D. thesis, MIT, 1999.

${ }^{9}$ P. Horsch and W. Stephan, Physica C 185, 1585 (1991); D. Poilblanc, Phys. Rev. B 49, 1477 (1994); see also Fig. 7 in Ref. 7.

${ }^{10}$ Details of this $p$-wave state will be published elsewhere.

${ }^{11}$ X. G. Wen, F. Wilczek, and A. Zee, Phys. Rev. B 39, 11413 (1989); P. A. Lee and N. Nagaosa, ibid. 46, 5621 (1992).

${ }^{12}$ G. B. Sha, D. A. Ivanov, P. A. Lee, and X.-G. Wen (unpublished). 